\section{Korean Cardiac Arrest Research Consortium (KoCARC): rationale, development, and implementation}

Joo Yeong Kim 1 , Sung Oh Hwang' ${ }^{2}$ Sang Do Shin ${ }^{3}$, Hyuk Jun Yang ${ }^{4}$, Sung Phil Chung ${ }^{5}$, Sung Woo Lee ${ }^{6}$, Kyung Jun Song ${ }^{3}$, Seung Sik Hwang ${ }^{7}$, Gyu Chong Cho ${ }^{8}$, Sung Woo Moon ${ }^{1}$, Kyuseok Kim ${ }^{9}$, Won Young $\mathrm{Kim}^{10}$, Seil $\mathrm{Oh}^{11}$, Young Ho Kwak ${ }^{3}$; on behalf of KoCARC

1Department of Emergency Medicine, Korea University Ansan Hospital, Korea University College of Medicine, Ansan, Korea

2Department of Emergency Medicine, Yonsei University Wonju College of Medicine, Wonju, Korea

3Department of Emergency Medicine, Seoul National University Hospital, Seoul, Korea

${ }^{4}$ Department of Emergency Medicine, Gachon University College of Medicine, Incheon, Korea

${ }^{5}$ Department of Emergency Medicine, Yonsei University College of Medicine, Seoul, Korea

${ }^{6}$ Department of Emergency Medicine, Korea University Anam Hospital, Korea University College of

Medicine, Seoul, Korea

${ }^{7}$ Department of Preventive Medicine, Seoul National University School of Public Health, Seoul, Korea

${ }^{8}$ Department of Emergency Medicine, Kang Dong Sacred Heart Hospital, Hallym University School of medicine, Seoul, Korea

${ }^{9}$ Department of Emergency Medicine, Seoul National University Bundang Hospital, Seongnam, Korea

${ }^{10}$ Department of Emergency Medicine, Asan Medical Center, University of Ulsan College of Medicine, Seoul, Korea

${ }^{11}$ Department of Internal Medicine, Seoul National University College of Medicine, Seoul, Korea

Objective This study aimed to describe the conceptualization, development, and implementation processes of the newly established Korean Cardiac Arrest Resuscitation Consortium (KoCARC) to improve out-of-hospital cardiac arrest (OHCA) outcomes.

Methods The KoCARC was established in 2014 by recruiting hospitals willing to participate voluntarily. To enhance professionalism in research, seven research committees, the Epidemiology and Preventive Research Committee, Community Resuscitation Research Committee, Emergency Medical System Resuscitation Research Committee, Hospital Resuscitation Research Committee, Hypothermia and Postresuscitation Care Research Committee, Cardiac Care Resuscitation Committee, and Pediatric Resuscitation Research Committee, were organized under a steering committee. The KoCARC registry was developed with variables incorporated in the currently existing regional OHCA registries and Utstein templates and were collected via a web-based electronic database system. The KoCARC study population comprises patients visiting the participating hospitals who had been treated by the emergency medical system for OHCA presumed to have a cardiac etiology.

Results A total of 62 hospitals volunteered to participate in the KoCARC, which captures 33.0\% of the study population in Korea. Web-based data collection started in October 2015, and to date (December 2016), there were 3,187 cases compiled in the registry collected from 32 hospitals.

Conclusion The KoCARC is a self-funded, voluntary, hospital-based collaborative research network providing high level evidence in the field of OHCA and resuscitation. This paper will serve as a reference for subsequent KoCARC manuscripts and for data elements collected in the study.

Keywords Out-of-hospital cardiac arrest; Cardiopulmonary resuscitation; Research
elSSN: 2383-4625

Received: 1 August 2017

Revised: 9 September 2017

Accepted: 24 September 2017

Correspondence to: Sung Oh Hwang Department of Emergency Medicine, Yonsei University Wonju College of Medicine, 20 Ilsan-ro, Wonju 26426, Korea

E-mail: shwang@yonsei.ac.kr

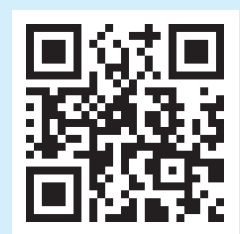

How to cite this article:

Kim JY, Hwang SO, Shin SD, Yang HJ, Chung SP, Lee SW, Song KJ, Hwang SS, Cho GC, Moon SW, Kim K, Kim WY, Oh S, Kwak YH; on behalf of KoCARC. Korean Cardiac Arrest Research Consortium (KoCARC): rationale, development, and implementation. Clin Exp Emerg Med 2018;5(3):165-176.

This is an Open Access article distributed under the terms of the Creative Commons Attribution Non-Commercial License (http:// creativecommons.org/licenses/by-nc/4.0/). 


Capsule
Summary is already known
$\begin{aligned} & \text { A regional research network and registries have been established to collect and pool data as a platform to produce evi- } \\ & \text { dence-based practices, to conduct large community or system-based intervention trials, and ultimately to produce } \\ & \text { meaningful research affecting policies in the area of out-of-hospital cardiac arrest resuscitation. }\end{aligned}$
What is new in the current study
In Korea, a hospital-based collaborative research network, the Korean Cardiac Arrest Research Consortium (KoCARC),
was organized with 62 hospitals participating voluntarily. The aim of the KoCARC is to coordinate various research
studies conducted on out-of-hospital cardiac arrest resuscitation, to strengthen the cooperative effort in these studies,
and to provide a stable data collecting system to all participating researchers.

\section{INTRODUCTION}

Out-of-hospital cardiac arrest (OHCA) is a major public health burden with a global average incidence among adults of 55 OHCAs per 100,000 person-years and an average survival rate of $7 \% .{ }^{1}$ In Korea, the survival rate is $3.0 \%$ with a good neurologic recovery rate of $0.9 \%$, which is lower than the global rate. ${ }^{2}$ For over 30 years, various efforts have been made to predict OHCA risk and outcome, ${ }^{3,4}$ but survival still varies between countries. ${ }^{5,6}$ In Korea, the trend of resuscitation performance and outcome was significantly different from urbanization levels. ${ }^{2}$ A missing link in the chain of survival exists and implementation of evidence-based practice to identify and strengthen the missing link is necessary.

Regional registries, such as the Cardiac Arrest Registry to Enhance Survival, ${ }^{7}$ the Canadian Ontario Prehospital Advanced Life Support network, ${ }^{4}$ and the Resuscitation Outcomes Consortium $(\mathrm{ROC})^{8}$ from North America have produced meaningful research affecting policies in the area of OHCA resuscitation. The PanAsian Resuscitation Outcome Study ${ }^{5}$ was established to derive best practices in the Asian region despite limited resources and heterogeneity across systems. The key property of these registries is the collection and pooling of data as a platform to produce evidence-based practices.

Considering limitations in sample size and resources needed for community- or system-based intervention trials, the concept of a large-scale collaborative research network has emerged. This research network-based registry enables researchers to analyze the risk and predictors of survival of OHCA and helps implement evidence-based strategies in resuscitation.

The aim of this article was to describe the conceptualization, implementation processes, potential benefit, and future plans for a newly established Korean resuscitation research network to improve OHCA outcomes.

\section{METHODS}

\section{Background and process}

The Korean Cardiac Arrest Resuscitation Consortium (KoCARC) is a collaborative research network developed to coordinate various research studies in the field of OHCA resuscitation and to strengthen the cooperative effort in conducting these studies. It was organized in 2014 by recruiting hospitals willing to participate voluntarily. In addition to emergency medicine, the KoCARC is expanding its research collaboration to include cardiology, preventive medicine, and epidemiology. To enhance the professionalism and effectiveness in research, seven research committees, the Epidemiology and Preventive Research Committee, Community Resuscitation Research Committee, Emergency Medical System Resuscitation Research Committee, Hospital Resuscitation Research Committee, Hypothermia and Postresuscitation Care Research Committee, Cardiac Care Resuscitation Committee, and Pediatric Resuscitation Research Committee, have been organized. In addition, the Data Safety and Monitoring Board Committee was organized to provide data quality management and the Security and Ethics Committee to oversee security and ethical issues in the research. Monthly meetings of the interdisciplinary steering committee, consisting of the consortium chair, steering committee chair, each research committee chair, and secretariat, are held to approve the proposed research agendas, support the research, manage the data-collecting system, and mediate between hospitals participating in multicenter trials. The organization of the KoCARC is shown in Fig. 1.

\section{Consensus building}

From December 2014 to September 2015, the Steering Committee developed the KoCARC registry data elements and definitions. The committee identified a comprehensive list of variables, performed consensus surveys, and identified the variables as core or supplemental. Core variables were confirmed via surveys at all partici- 


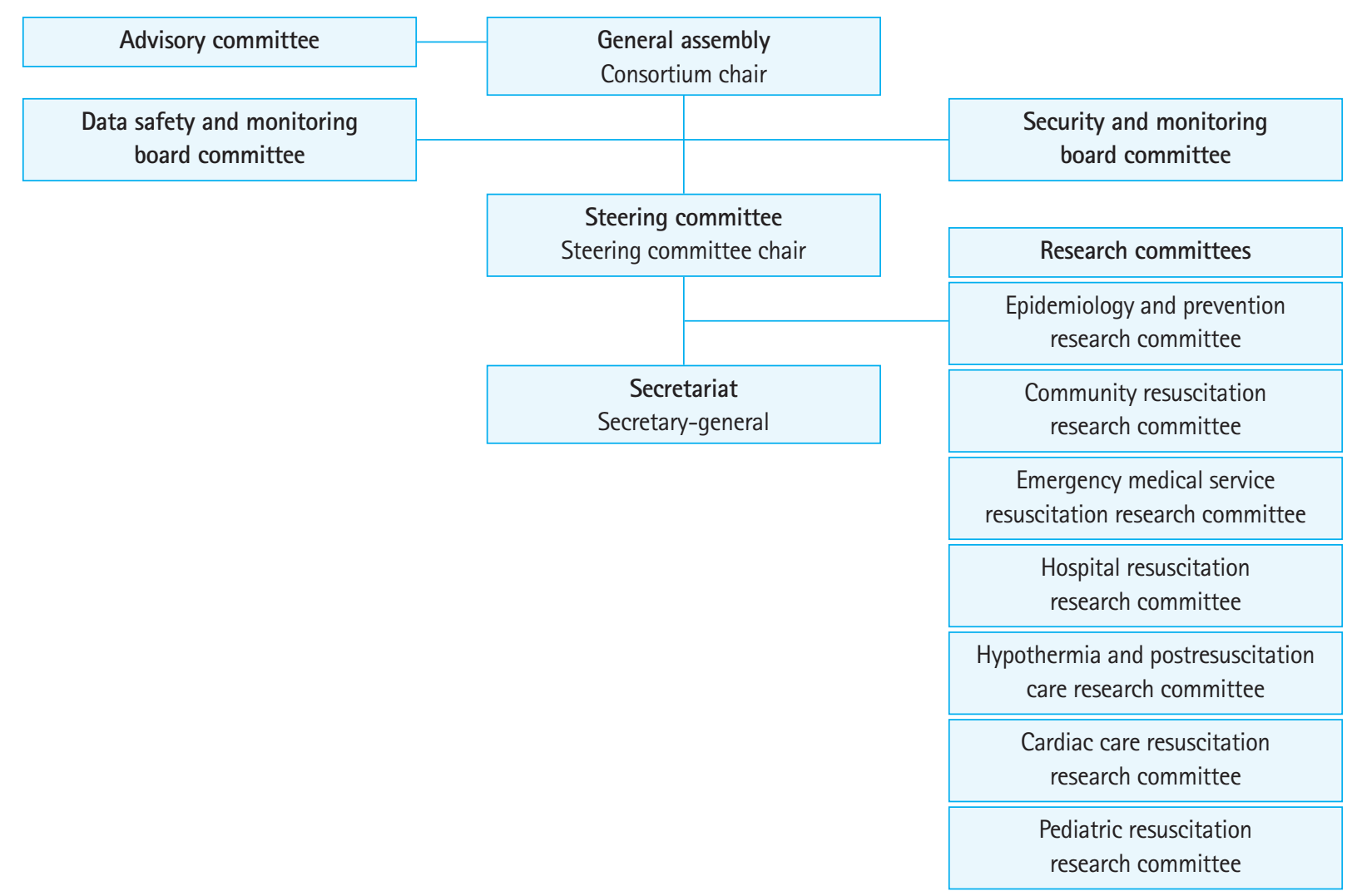

Fig. 1. Organization of Korean Cardiac Arrest Resuscitation Consortium.

pating hospitals asking about the feasibility of collecting the data. Definitions and response options were developed for each variable incorporated in the currently existing regional OHCA registries, national OHCA registry, and the Utstein templates ${ }^{9}$ to achieve uniform reporting. The KoCARC registry is a data-collecting system composed of OHCA risk and prognostic factors developed to provide a platform for research conducted in the KoCARC.

\section{RESULTS}

\section{Concepts of the KoCARC}

The KoCARC is a hospital-based collaborative research network comprising multiple research committees on the platform of the KoCARC registry. It was developed to enhance the effectiveness and professionalism of research to produce evidence to strengthen the chain of survival.

\section{Implementation processes}

Registry data and collection protocol

Inclusion/exclusion criteria

The KoCARC registry was designed to include patients with OHCA transported to the participating emergency departments (ED) by emergency medical services (EMSs) with resuscitation efforts and patients who had a medical etiology identified by emergency physicians in each ED. The registry excluded OHCA patients with a terminal illness documented by medical records, patients under hospice care, pregnant patients, and patients with a previously documented 'Do Not Resuscitate' card. OHCA of definite nonmedical etiology, including trauma, drowning, poisoning, burn, asphyxia, or hanging, were also excluded. For patients younger than 19 years (age range defined as pediatric by the Korean Pediatric Society), OHCA patients transported to an ED by any method and with any etiology were included in the registry.

Data collection and quality assurance

Data are collected via a standardized registry form and entered into a web-based electronic database registry. Outliers or incorrect values are primarily filtered by this data entry system. Furthermore, each participating ED has a designated local research coordinator who is responsible for ensuring the accuracy of the data and verifying records. To maintain personal confidentiality, no patient identifiers appear on the website and each case is assigned a unique identifier based on the ED.

The quality management committee (OMC), consisting of emergency physicians, statistical experts, local research coordinators, and investigators in each $E D$, is organized before joining the proj- 
ect. The data dictionary and protocol for data entry were distributed to site coordinators before the actual data collection. The QMC quality assurance plan includes checking completeness and consistency of mandatory fields as well as checking for inbuilt validation rules that cross-check data fields. In addition, the $\mathrm{QMC}$ provides feedback regarding quality management processes to the research coordinators and investigators at the quarterly meetings. The research coordinator consults the $\mathrm{OMC}$ via e-mail for clarification

Table 1. KoCARC core variables by outcome and research field

\begin{tabular}{|c|}
\hline KoCARC core variable \\
\hline Patient information and outcome \\
\hline Date/time of ED visit \\
\hline Age/sex/name (initials)/date of birth \\
\hline ED direct visit or transfer from other hospital \\
\hline Result of ED resuscitation \\
\hline Result of ED disposition \\
\hline Date/time of resuscitation withdrawal \\
\hline Date/time of any ROSC/sustained ROSC/ED death/ED discharge/admission \\
\hline Mental status at admission \\
\hline Result of disposition after hospital discharge \\
\hline Result of neurologic recovery at hospital discharge \\
\hline Result of survival/neurologic recovery at 6 months from cardiac arrest \\
\hline Date/time of hospital discharge/death after admission \\
\hline Epidemiology and preventive medicine \\
\hline $\begin{array}{l}\text { Family history of sudden cardiac arrest/acute coronary syndrome/ } \\
\text { cerebrovascular accident/arrhythmia }\end{array}$ \\
\hline Smoking history/alcohol consumption history and frequency \\
\hline Medical history of hypertension/diabetes/dyslipidemia and treatment \\
\hline Community resuscitation \\
\hline Date/time of incident \\
\hline Witness and by whom \\
\hline Arrest location \\
\hline Bystander CPR/AED \\
\hline Public AED rhythm \\
\hline EMS resuscitation \\
\hline $\begin{array}{l}\text { Date/time of call received at dispatch center/ambulance arrived at scene/ } \\
\text { ambulance left scene }\end{array}$ \\
\hline First EMS ECG rhythm \\
\hline Advanced airway/drug used by EMS/EMS defibrillation \\
\hline ROSC at scene \\
\hline Date/time of EMS defibrillation/return of spontaneous circulation at scene \\
\hline Hospital resuscitation \\
\hline Return of spontaneous circulation at initially visited hospital (if transferred) \\
\hline $\begin{array}{l}\text { Date/time of return of spontaneous circulation at initial hospital visit } \\
\text { (if transferred) }\end{array}$ \\
\hline Initial ECG rhythm on arrival at ED \\
\hline Amount of epinephrine used until cessation of resuscitation/sustained ROSC \\
\hline Number of defibrillations performed in ED \\
\hline Requested/performed ECMO \\
\hline Provided mechanical CPR/ETCO2 monitor \\
\hline
\end{tabular}

(Continued to the next)
Table 1. Continued

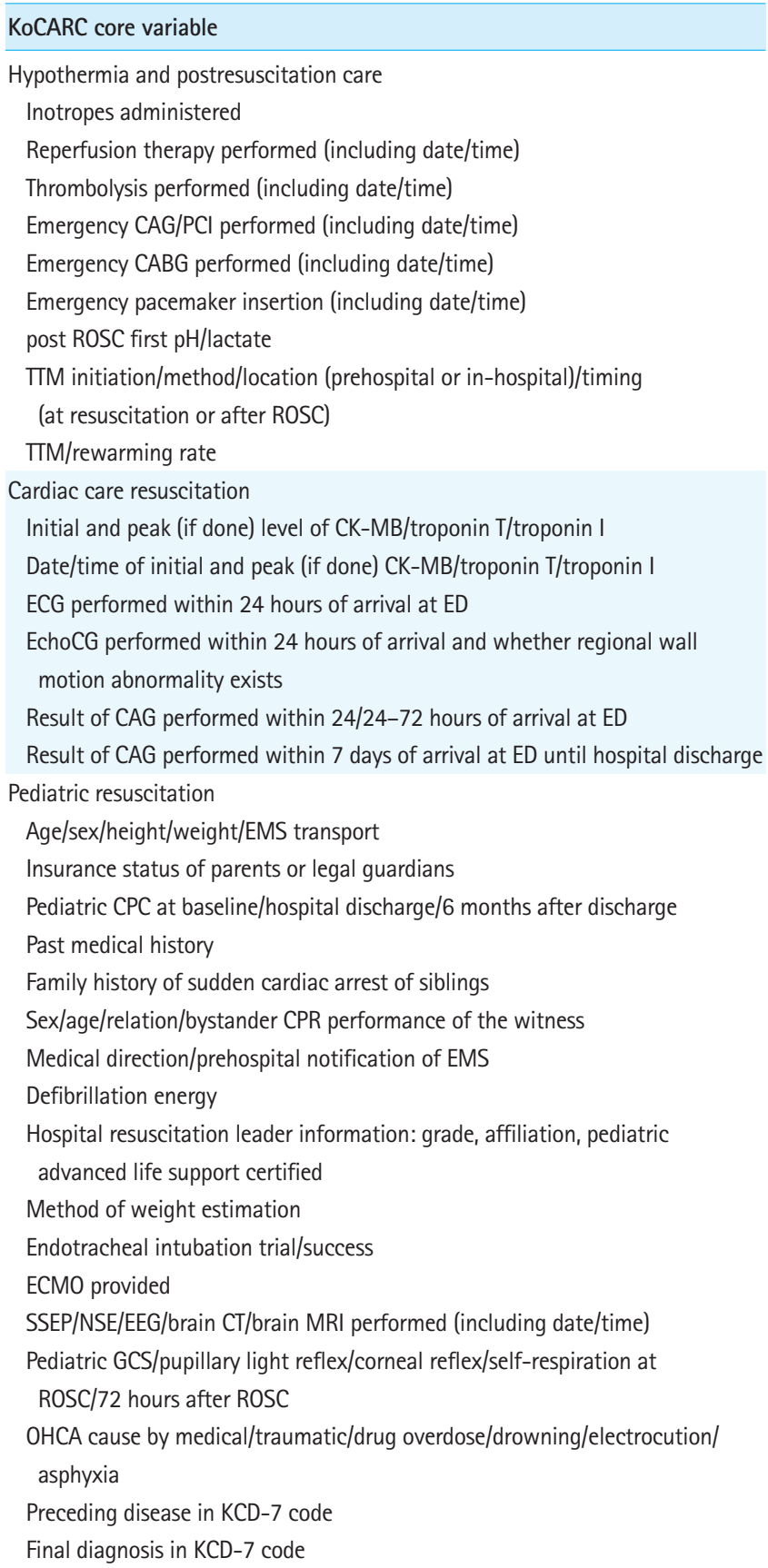

KoCARC, Korean Cardiac Arrest Resuscitation Consortium; ED, emergency de-

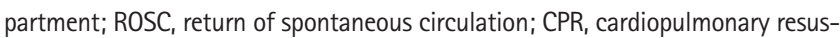
citation; AED, automated external defibrillator; EMS, emergency medical service; ECG, electrocardiography; ECMO, extracorporeal membrane oxygenation; $\mathrm{ETCO}_{2}$, end-tidal carbon dioxide; $\mathrm{CAG}$, coronary angiography; $\mathrm{PCl}$, percutaneous coronary intervention; $C A B G$, coronary artery bypass graft; $\Pi M$, target temperature management; $\mathrm{CK}-\mathrm{MB}$, creatine kinase $\mathrm{MB} ; \mathrm{CPC}$, cerebral performance category; SSEP, somatosensory evoked potential; NSE, neuron specific enolase; EEG, electroencephalography; $\mathrm{CT}$, computed tomography; $\mathrm{MRI}$, magnetic resonance imaging; GCS, Glasgow coma scale; KCD, Korean Standard Classification of Disease. 
when they are unable to objectively define coding elements.

Epidemiologic, community, and EMS information is collected by interviewing family members and ambulance crews. Information not obtained via interview is collected from the Emergency Medical Service Cardiac Registry. Research coordinators at each participating ED gather laboratory test and cardiac intervention results via medical record review. For survivors, the prognosis after 6 months from the event is assessed through telephone interviews with the survivors or their family members.

\section{Human subjects ethical issues}

The KoCARC data collecting protocol was reviewed and approved by each participating hospital's institutional review board (IRB). Most IRBs waived informed consent, but some required informed consent to use personal information such as telephone numbers used for the 6-month follow-up survey of neurologic recovery. Some hospitals required informed consent for pediatric resuscitation information.

\section{Variables measured}

The KoCARC registry consists of seven research fields categorized by OHCA risk and prognostic factors. Each field has core and optional variables. Core variables are listed in Table 1.

\section{Core variables by research field}

Epidemiology and preventive medicine

The family history of cardiovascular disease and sudden cardiac arrest is collected up to second-degree family members through family member interview or medical record review. Health behaviors, including smoking and alcohol drinking, and medical history including hypertension, diabetes mellitus, dyslipidemia and their treatment methods are also collected.

\section{Community resuscitation}

The time of OHCA occurrence, witness status, place of arrest, initial arrest electrocardiography (ECG) rhythm, and bystander cardiopulmonary resuscitation (CPR) information is collected through medical record review or from interviews of the ambulance crew. The initial arrest ECG rhythm is defined as the rhythm recorded in the public automatic external defibrillator if one is used. Otherwise, the initial arrest rhythm is primarily the initial EMS-monitored rhythm.

\section{EMS resuscitation}

EMS variables, including EMS response interval variables, EMS first monitored ECG rhythm, and types of advance procedures performed, are collected via the EMS Cardiac Arrest Registry or ambulance crew interviews. Prehospital return of spontaneous circulation (ROSC) is collected as a prehospital outcome variable.

Hospital resuscitation

The amount of epinephrine used throughout ED resuscitation, initial hospital ECG, and initiation of extracorporeal membrane oxygenation (ECMO) is collected through medical record review. Information regarding ROSC in OHCA patients transferred from other hospitals is also collected.

Hypothermia and postresuscitation care

Timing and methods of coronary reperfusion therapy and target temperature management (TTM) to enhance survival and prognosis after ROSC is collected via medical record review.

Cardiac care resuscitation

Laboratory test and coronary angiography results are collected. For laboratory results, initial and peak values (if done) of creatine kinase $\mathrm{MB}$, troponin I, and troponin T are collected. Coronary angiography results include the anatomic lesion and type of stenosis.

\section{Pediatric resuscitation}

Information is collected on pediatric risk factors including premature birth, medical history, baseline cerebral performance category (CPC), family history of sudden cardiac arrest of sibling, participating physician's level at ED resuscitation, presumed etiology of cardiac arrest, result of prognostic tests after ROSC, and outcome. The 6-month follow-up CPC is also collected via telephone interview after IRB approval.

\section{Outcome measures}

The outcome data collected are survival to hospital discharge and neurologic recovery at hospital discharge. Hospital outcome includes date and time of death or discharge. Neurologic status is assessed using CPC with good neurologic recovery defined as CPC 1 or 2 . The neurologic status of survivors after 6 months from the arrest event is also collected.

\section{Sample size and planned data analysis}

The sample size for each potential risk factor evaluated for association with better survival will be computed based on the study proposal with a type I error of 0.05 and power of $80 \%$ or $90 \%$. Participating sites (or investigators) will be provided with preliminary estimates of risk factors and predictors for outcomes from data collected for the purpose of planning the research. Data analysis will be performed based on the study proposal established during the study planning stage. 


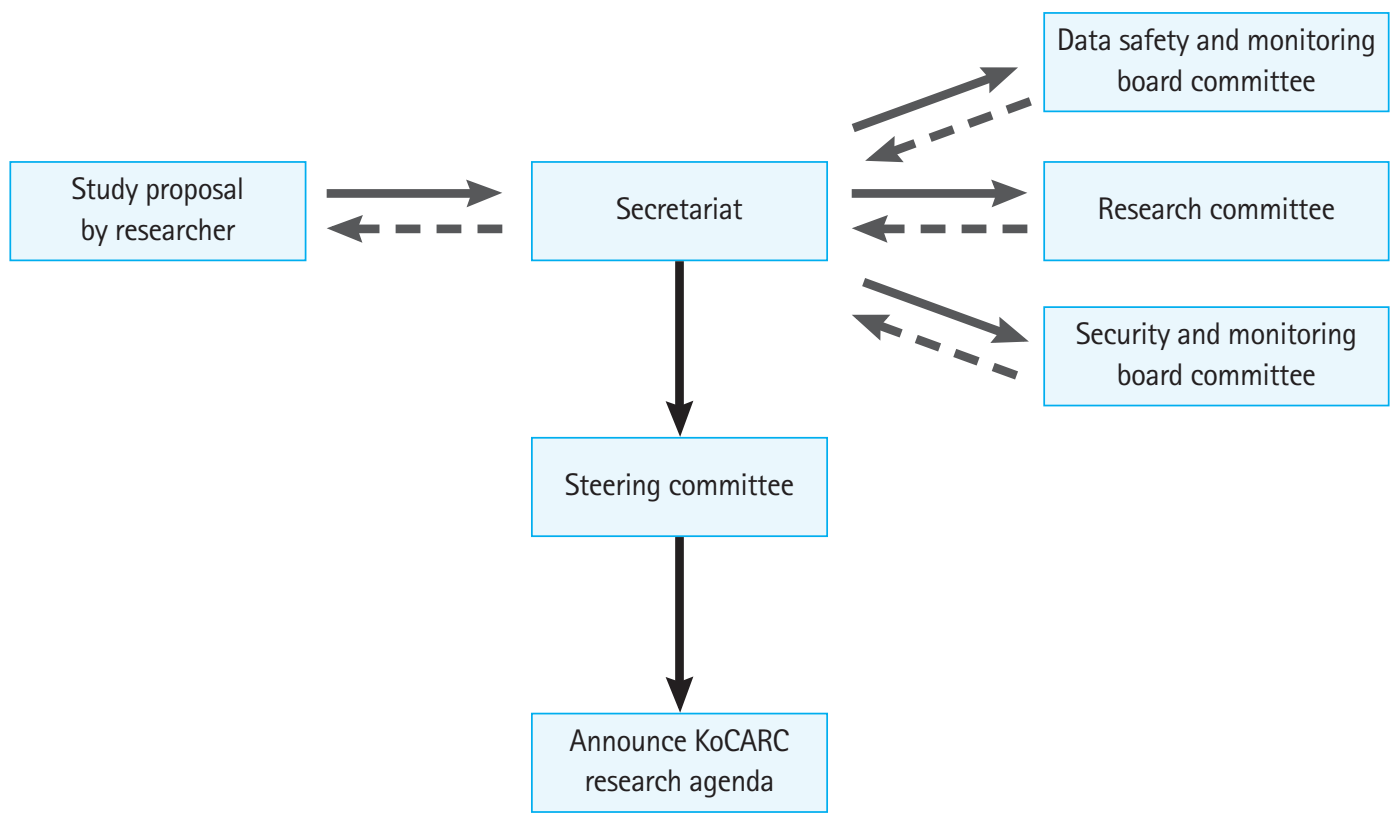

Fig. 2. Flow chart of study proposal to be accepted as an official Korean Cardiac Arrest Resuscitation Consortium (KoCARC) research agenda.

Table 2. Numbers of cardiac-etiology OHCA patients transported to KoCARC participating hospitals in 2015 according to national OHCA data (provided by the KCDC)

\begin{tabular}{lrr}
\hline Administrative region & All & \multicolumn{1}{c}{ KoCARC } \\
\hline All & 22,079 & $7,293(33.0)$ \\
Seoul & 3,889 & $2,272(58.4)$ \\
Busan & 1,475 & $265(18.0)$ \\
Daegu & 1,009 & $490(48.6)$ \\
Incheon & 1,206 & $574(47.6)$ \\
Gwangju & 514 & $106(20.6)$ \\
Daejeon & 647 & $217(33.5)$ \\
Ulsan & 379 & $76(20.1)$ \\
Gyeonggi & 4,491 & $1,950(43.4)$ \\
Gangwon & 1,013 & $412(40.7)$ \\
Chungbuk & 832 & $83(10.0)$ \\
Chungnam & 1,042 & $195(18.7)$ \\
Jeonbuk & 942 & $149(15.8)$ \\
Jeonnam & 1,067 & $0(0.0)$ \\
Gyeongbuk & 1,550 & $101(6.5)$ \\
Gyeongnam & 1,522 & $291(19.1)$ \\
Jeju & 463 & $112(24.2)$ \\
\hline
\end{tabular}

Values are presented as number or number (\%).

OHCA, out-of-hospital cardiac arrest; KoCARC, Korean Cardiac Arrest Resuscitation Consortium; KCDC, Korean Centers for Disease Control and Prevention.

Descriptive analyses comparing outcomes of patients exposed or not exposed to risk factors will be reported using the chisquare test or Wilcoxon rank-sum test as appropriate. The categories with sparse data will be grouped together in biologically meaningful ways. The main data analysis will be performed based on the study hypothesis. Regarding certain outcome variables

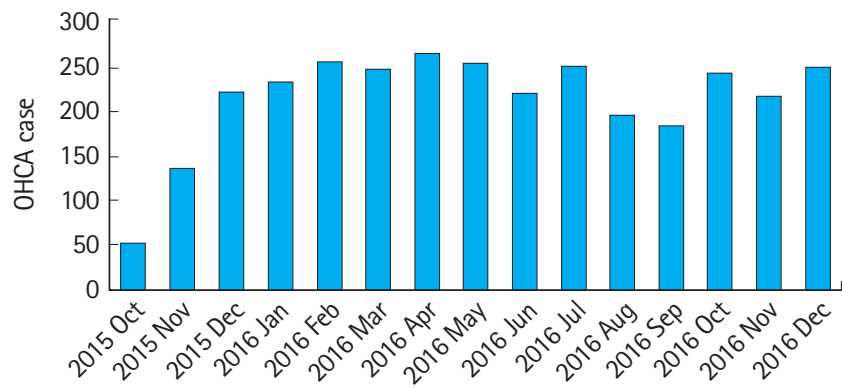

Fig. 3. Data collection by month. OHCA, out-of-hospital cardiac arrest.

such as survival to discharge, good neurologic outcome, or 6-month survival, multivariate logistic regression will be performed with a priori selection of clinically important covariates.

\section{Study proposal process}

To qualify as a research network and to improve the level of evidence in the field of cardiac arrest and/or CPR, the KoCARC recruits research subjects from individual researchers or participating hospitals. Any researcher willing to conduct research submits a study proposal to the secretariat as appropriate for the study method (Appendices 1 and 2). The secretariat reviews the study proposal for completeness of the essential information and provides feedback to the researchers. After the secretariat review, the proposal is sent to each appropriate research committee, the Data Safety and Monitoring Board Committee, the Security and Ethics Committee, and finally to the Steering Committee for final approval. A selected research subject is announced as an official 
Table 3. Characteristics of KoCARC data from October 2015 to December 2016

\begin{tabular}{lc}
\hline Characteristics & Value \\
\hline All & $3,187(100.0)$ \\
Sex, male & $2,073(65.0)$ \\
Age & $69(55-79)$ \\
Witness & $1,924(60.4)$ \\
Witnessed or found by layperson & $2,776(87.1)$ \\
Arrest at public place & $672(21.1)$ \\
Bystander CPR & $1,553(48.7)$ \\
Bystander AED applied & $70(2.2)$ \\
Prehospital shockable ECG & $579(18.2)$ \\
Call to scene interval & $7(5-10)$ \\
Scene Resuscitation interval & $11(7-17)$ \\
Scene to hospital interval & $10(7-15)$ \\
Survival to discharge & $365(11.5)$ \\
Good neurologic recovery (CPC 1-2) & $248(7.8)$ \\
\hline
\end{tabular}

Values are presented as number (\%) or median (lower quartile-upper quartile). KoCARC, Korean Cardiac Arrest Resuscitation Consortium; CPR, cardiopulmonary resuscitation; $A E D$, automated external defibrillator; $E C G$, electrocardiography; CPC, cerebral performance category.

research agenda of the KoCARC to all participating researchers. The process of the study proposal accepted as an official KoCARC study agenda is shown in Fig. 2.

\section{Progress to date}

Sixty-two hospitals from all provinces except Jeonnam Province volunteered to participate in the KoCARC. All participating EDs are designated as local or regional emergency medical centers which constitute the top two levels of EDs legislated by law. National OHCA data collected by the Korean Centers for Disease Control and Prevention show that 33.0\% of EMS-treated patients with OHCA of cardiac etiology are captured in the registry. OHCA patients transported to KoCARC participating hospitals located in metropolitan cities such as Seoul, Daegu, and Incheon comprise $58.4 \%, 48.6 \%$, and $47.6 \%$ of all cardiac etiology OHCA cases. The proportion of the study population captured in participating hospitals in 2015 is shown in Table 2.

Web-based data collection started in October 2015, and by December 2016, 32 hospitals had enrolled 3,187 cases in the KoCARC registry. Regarding the first 2 months as the protocol implementation period, 200 to 250 cases per month were consistently captured throughout 2016, as shown in Fig. 3.

Characteristics of the initially collected KoCARC data are shown in Table 3. Among the 3,187 OHCA cases, bystander CPR was performed in $48.7 \%$ and a public access automatic external defibrillator was applied in $2.2 \%$ of cases. Furthermore, $11.5 \%$ of patients survived to discharge and $7.8 \%$ recovered with a good neurologic outcome. Because most of the participating hospitals are regional
Table 4. In-hospital intervention performed for OHCA patients who survived to admission

\begin{tabular}{lc}
\hline In-hospital intervention & Admitted OHCA \\
\hline All & $841(100.0)$ \\
TPA thrombolysis & $91(10.8)$ \\
PCI performed & $124(14.7)$ \\
CABG performed & $10(1.2)$ \\
Pacemaker inserted & $13(1.5)$ \\
ICD inserted & $27(3.2)$ \\
TM performed & $274(32.6)$
\end{tabular}

Values are presented as number (\%).

OHCA, out-of-hospital cardiac arrest; TPA, tissue plasminogen activator; $\mathrm{PCl}$, percutaneous coronary intervention; $C A B G$, coronary artery bypass graft; $I C D$, implantable cardioverter defibrillator; $T \mathrm{M}$, target temperature management.

or local emergency medical centers that provide high-quality resuscitation and comprehensive postresuscitation care, survival outcome is higher than has been previously reported.

Table 4 shows in-hospital interventions provided for patients surviving to admission. Among admitted patients, primary percutaneous coronary intervention (PCI) was performed in 14.7\% and TाM in $32.6 \%$ of patients.

\section{Potential benefit and future plans}

The KoCARC is a volunteer-based, low-cost model of a research network that will provide long-term data needed to perform research to improve OHCA survival. Ultimately, this platform system will facilitate research using the infrastructure provided by the consortium to conduct studies to strengthen not yet proven but critical links in the chain of survival. We are planning to implement the proposed agendas and concurrently perform randomized controlled trials to address important epidemiologic and clinical questions regarding cardiac arrest. The findings resulting from such research may influence and improve public health.

\section{DISCUSSION}

Currently, there is little evidence from randomized controlled trials to lead us to the best strategy for improving outcomes of OHCA. ${ }^{10}$ Awareness of such issues emphasizes the importance of large-scale research networks and the importance of uniformly collected data, which can objectively compare the effects of resuscitation interventions.

To the best of our knowledge, this is the largest volunteerbased research network of OHCA in Korea. Currently, in December 2016, 62 hospitals have expressed interest in participating in KoCARC and 32 hospitals are actively inputting data. Throughout 15 months of data collection, we have been able to acquire highquality information on the risk, care, process, and outcome of 


\section{3,187 unique OHCA cases.}

The fact that the KoCARC registry provides the platform and infrastructure necessary to acquire the evidence to identify the missing links in the chain of survival makes it equal in significance to the ROC. ${ }^{8}$ However, compared with the diverse regional EMS and dispatch systems and diverse organizations that input data to the ROC database, the KoCARC collects prehospital variables from the uniform fire-based EMS system, and data are registered on a hospital basis. In Korea, as prehospital termination of resuscitation is not legally possible, all OHCA cases included in the KoCARC are transported to hospitals. Therefore, the hospitalbased data collection system of the KoCARC is the most efficient and feasible method to capture cases. Furthermore, each participating hospital has a designated local research coordinator responsible for data collection and participates in quality assurance of collected data.

It is well-known that high-quality CPR at the community and EMS level is important for increasing survival of OHCA. ${ }^{11-13}$ Recently, emphasis has been put on the fifth chain of survival, postresuscitation care. Research demonstrating that active postresuscitation care improves neurologic outcomes ${ }^{14}$ has been published, and the American Heart Association guidelines have been updated to recommend selecting a temperature between $32^{\circ} \mathrm{C}$ and $36^{\circ} \mathrm{C}$ for comatose post ROSC patients compared with $32^{\circ} \mathrm{C}$ to $34^{\circ} \mathrm{C}$ in the 2010 guidelines. ${ }^{15}$ Active emergent $\mathrm{PCl}$ for OHCA survivors without ST elevation has shown favorable neurologic outcomes $^{16}$ and a delayed arrest to $\mathrm{PCl}$ interval was associated with poor survival outcomes after OHCA. ${ }^{17}$ Previous OHCA registries, which had the goal of public health surveillance, has limitations in collecting in-depth in-hospital procedure data except for hospital outcomes. The Cardiac Arrest Registry to Enhance Survival collects five data elements from hospitals: ED outcome, receiving hospital outcome, patient disposition, CPC at discharge, simple measurement of functional status, and use of hypothermia. ${ }^{7}$ The Pan-Asian Resuscitation Outcome Study collects hospital outcome as a core variable, but hospital resuscitation and intervention are collected as optional variables. ${ }^{5}$ The KoCARC, on the other hand, is a research-based registry and prospectively collects data on intra-CPR interventions such as ECMO, mechanical CPR, and rates of epinephrine use along with postresuscitation data including the method and timing of coronary reperfusion therapy and detailed information on the method and timing of hypothermia management as core variables. All of these data will allow investigation of outcomes based on interventions. Furthermore, as the vast majority of the participating hospitals are capable of providing comprehensive postresuscitation care, the KoCARC will collect in-depth and high-quality data on hospital intervention.
There is growing interest in the long-term outcomes of patients surviving OHCA. Studies have reported that CPC at hospital discharge was associated with long-term survival outcomes ${ }^{18-20}$ and that neurologic recovery was rare in patients with CPC 3 or 4 more than 6 months after cardiac arrest. ${ }^{21}$ Several studies have evaluated long-term outcomes of OHCA survivors using a small sample or one center. ${ }^{22-24}$ However, the KoCARC collects data on the neurologic status of OHCA patients 6 months after cardiac arrest by telephone interview with survivors or their primary caregivers. Although IRB approval at each participating site will be needed, it will be meaningful to conduct long-term follow-up of OHCA survivors on a multicenter scale.

The current study has several limitations. First, indications for applying in-hospital interventions, such as ECMO, $\mathrm{TM}$, or $\mathrm{PCl}$, are not standardized between the participating hospitals. Although most of the hospitals may follow the current CPR guidelines, limitations of resources or patient/guardian characteristics affect treatment intensity. Second, missing or incomplete data or unrecognized variables may confound the results. We tried to minimize this risk by implementing quality assurance data checks built into the data entry system and also by off-line periodic quality assurance meetings with local designated coordinators. However, there are still missing values for some variables. Third, the IRB approval status of each hospital is different such that collection of data on pediatric resuscitation or 6-month follow-up data is limited in some hospitals. This may lead to incomplete data for some cases. Finally, like all observational studies, causal inference cannot be established, but only associations between risk factors and outcomes. Cautious interpretation is needed for the results. However, if observational studies are carefully conducted, the estimated effects may be consistent with the results of clinical trials.

In conclusion, the KoCARC is a voluntary, hospital-based collaborative research network that has constructed a stable data collecting system that provides a platform for investigating the missing links in the chain of survival. This article will serve as a reference for subsequent KoCARC manuscripts and for the core data elements collected in the study.

\section{CONFLICT OF INTEREST}

No potential conflict of interest relevant to this article was reported.

\section{ACKNOWLEDGMENTS}

This consortium was supported by the Korean Centers for Disease Control and Prevention during the organizing stage. Currently, 
KoCARC is partly supported by the Korean Association of Cardiopulmonary Resuscitation.

We would like to acknowledge and thank to members of Secretariat: Jeong Ho Park (Seoul National University hospital), Sun Young Lee (Seoul National University hospital), Jung Eun Kim (Seoul National University hospital), Na Young Kim (Seoul National University hospital), Min Ji Kwon (Seoul National University hospital); and to investigators from all participating hospitals in KoCARC: Myoung Chun Kim (Kyung Hee University Hospital at Gangdong), Sang Kuk Han (Kangbuk Samsung Medical Center), Kwang Je Baek (Konkuk University Medical Center), Han Sung Choi (Kyung Hee University Hospital), Sung Hyuk Choi (Korea University Guro Hospital), Ik Joon Jo (Samsung Medical Center), Jong Whan Shin (SMGSNU Boramae Medical Center), Sang Hyun Park (Seoul Medical Center), In Cheol Park (Yonsei University Severance Hospital), Chul Han (Ewha Womans University Mokdong Hospital), Chu Hyun Kim (Inje University Seoul Paik Hospital), Gu Hyun Kang (Hallym University Kangnam Sacred Heart Hospital), Tai Ho Im (Hanyang University Seoul Hospital), Seok Ran Yeom (Pusan National University Hospital), Jae Hoon Lee (Dong-A University Hospital), Ha Young Park (Inje University Haeundae Hospital), Jeong Bae Park (Kyungpook National University Hospital), Sung Jin Kim (Keimyung University Dongsan Medical Center), Kyung Woo Lee (Daegu Catholic University Medical Center), Woon Jeong Lee (The Catholic University of Korea Incheon ST. Mary's Hospital), Sung Hyun Yun (Catholic Kwandong University), Ah Jin Kim (Inha University Hospital), Kyung Woon Jeong (Chonnam National University Hospital), Sun Pyo Kim (Chosun University Hospital), Jin Woong Lee (Chungnam National University Hospital), Sung Soo Park (Konyang University Hospital), Ryeok Ahn (Konyang University Hospital), Kyoung Ho Choi (The Catholic University of Korea Uijeongbu ST. Mary's Hospital), Young Gi Min (Ajou University Hospital), In Byung Kim (Myongji Hospital), Ji Hoon Kim (The Catholic University of Korea Buchen ST.Mary's Hospital), Seung Chul Lee (Dongguk University Ilsan Hospital), Young Sik Kim (Bundang Jesaeng General Hospital), Hun Lim (Soonchunhyang University Bucheon Hospital), Jin Sik Park (Sejong Hospital), Jun Seok Park (Inje University llsan Paik Hospital), Dai Han Wi (Wonkwang University Sanbon Hospital), Ok Jun Kim (Cha University Bundang Cha Hospital), Bo Seung Kang (Hanyang University Guri Hospital), You Dong Sohn (Hallym University Pyeongchon Sacred Heart Hospital), Soon Joo Wang (Hallym University Dongtan Sacred Heart Hospital), Se Hyun Oh (GangNeung Asan Hospital), Jun Hwi Cho (Kangwon National University Hospital), Mu Eob An (Hallym University Chuncheon Sacred Heart Hospital), Ji Han Lee (Chungbuk National University Hospital), Han Joo Choi (Dankook University Hospital), Jung Won Lee (Soonchunhyang University Cheonan Hospital), Tae Oh Jung (Chonbuk National University Hospital), Dai
Hai Choi (Dongguk University Gyeongju Hospital), Seong Chun Kim (Gyeongsang National University Hospital), Ji Ho Ryu (Pusan National University Yangsan Hospital ), Won Kim (Cheju Halla General Hospital), Sung Wook Song (Jeju National University Hospital).

\section{REFERENCES}

1. Berdowski J, Berg RA, Tijssen JG, Koster RW. Global incidences of out-of-hospital cardiac arrest and survival rates: systematic review of 67 prospective studies. Resuscitation 2010;81:147987.

2. Ro YS, Shin SD, Song KJ, et al. A trend in epidemiology and outcomes of out-of-hospital cardiac arrest by urbanization level: a nationwide observational study from 2006 to 2010 in South Korea. Resuscitation 2013;84:547-57.

3. Herlitz J, Ekstrom L, Wennerblom B, Axelsson A, Bang A, Holmberg $S$. Prognosis among survivors of prehospital cardiac arrest. Ann Emerg Med 1995;25:58-63.

4. Stiell IG, Wells GA, DeMaio VJ, et al. Modifiable factors associated with improved cardiac arrest survival in a multicenter basic life support/defibrillation system: OPALS Study Phase I results. Ontario Prehospital Advanced Life Support. Ann Emerg Med 1999;33:44-50.


Outcomes Study (PAROS): rationale, methodology, and implementation. Acad Emerg Med 2011;18:890-7.

6. Grasner JT, Lefering R, Koster RW, et al. EuReCa ONE-27 Nations, ONE Europe, ONE Registry: a prospective one month analysis of out-of-hospital cardiac arrest outcomes in 27 countries in Europe. Resuscitation 2016;105:188-95.

7. McNally $B$, Stokes $A$, Crouch A, Kellermann AL; CARES Surveillance Group. CARES: Cardiac Arrest Registry to Enhance Survival. Ann Emerg Med 2009;54:674-83.

8. Morrison $\sqcup$, Nichol G, Rea TD, et al. Rationale, development and implementation of the Resuscitation Outcomes Consortium Epistry-Cardiac Arrest. Resuscitation 2008;78:161-9.

9. Perkins GD, Jacobs IG, Nadkarni VM, et al. Cardiac arrest and cardiopulmonary resuscitation outcome reports: update of the Utstein resuscitation registry templates for out-of-hospital cardiac arrest. A statement for healthcare professionals from a task force of the International Liaison Committee on Resuscitation (American Heart Association, European Resuscitation Council, Australian and New Zealand Council on Resuscitation, Heart and Stroke Foundation of Canada, InterAmerican Heart Foundation, Resuscitation Council of Southern Africa, Resuscitation Council of Asia); and the American Heart Association Emergency Cardiovascular Care Committee and 
the Council on Cardiopulmonary, Critical Care, Perioperative and Resuscitation. Resuscitation 2015;96:328-40.

10. Nichol G, Steen P, Herlitz J, et al. International Resuscitation Network Registry: design, rationale and preliminary results. Resuscitation 2005;65:265-77.

11. Cummins RO, Eisenberg MS, Hallstrom AP, Litwin PE. Survival of out-of-hospital cardiac arrest with early initiation of cardiopulmonary resuscitation. Am J Emerg Med 1985;3:114-9.

12. Margey R, Browne L, Murphy E, et al. The Dublin cardiac arrest registry: temporal improvement in survival from out-of-hospital cardiac arrest reflects improved pre-hospital emergency care. Europace 2011;13:1157-65.

13. Hansen $C M$, Kragholm $K$, Granger $C B$, et al. The role of bystanders, first responders, and emergency medical service providers in timely defibrillation and related outcomes after out-of-hospital cardiac arrest: results from a statewide registry. Resuscitation 2015;96:303-9.

14. Kim JY, Shin SD, Ro YS, et al. Post-resuscitation care and outcomes of out-of-hospital cardiac arrest: a nationwide propensity score-matching analysis. Resuscitation 2013;84:1068-77.

15. Callaway CW, Donnino MW, Fink EL, et al. Part 8: post-cardiac arrest care. 2015 American Heart Association Guidelines Update for Cardiopulmonary Resuscitation and Emergency Cardiovascular Care. Circulation 2015;132(18 Suppl 2):S465-82.

16. Dumas F, Bougouin W, Geri G, et al. Emergency percutaneous coronary intervention in post-cardiac arrest patients without ST-segment elevation pattern: insights from the PROCAT II Registry. JACC Cardiovasc Interv 2016;9:1011-8.
17. Jeong J, Ro YS, Shin SD, Song KJ, Hong KJ, Ahn KO. Association of time from arrest to percutaneous coronary intervention with survival outcomes after out-of-hospital cardiac arrest. Resuscitation 2017;115:148-54.

18. Hsu CH, Li J, Cinousis MJ, et al. Cerebral performance category at hospital discharge predicts long-term survival of cardiac arrest survivors receiving targeted temperature management. Crit Care Med 2014;42:2575-81.

19. Phelps R, Dumas F, Maynard C, Silver J, Rea T. Cerebral Performance Category and long-term prognosis following out-ofhospital cardiac arrest. Crit Care Med 2013;41:1252-7.

20. Rittenberger JC, Raina K, Holm MB, Kim YJ, Callaway CW. Association between Cerebral Performance Category, Modified Rankin Scale, and discharge disposition after cardiac arrest. Resuscitation 2011;82:1036-40.

21. Kim YJ, Ahn S, Sohn CH, et al. Long-term neurological outcomes in patients after out-of-hospital cardiac arrest. Resuscitation 2016;101:1-5.

22. Lundgren-Nilsson A, Rosen $H$, Hofgren $C$, Sunnerhagen KS. The first year after successful cardiac resuscitation: function, activity, participation and quality of life. Resuscitation 2005;66:2859.

23. Raina KD, Rittenberger JC, Holm MB, Callaway CW. Functional outcomes: one year after a cardiac arrest. Biomed Res Int 2015;2015:283608.

24. Lim C, Verfaellie M, Schnyer D, Lafleche G, Alexander MP. Recovery, long-term cognitive outcome and quality of life following out-of-hospital cardiac arrest. J Rehabil Med 2014;46:691-7. 
Joo Yeong Kim, et al.

Appendix 1. Study proposal form of observational study

\begin{tabular}{|c|c|c|}
\hline Item & & Observational study content \\
\hline Title & & \\
\hline Proposer & & \\
\hline Co-workers & & \\
\hline Institution & & \\
\hline Study design & $\begin{array}{l}\square \text { Cohort study } \\
\square \text { Case-control study } \\
\square \text { Case-series study } \\
\square \text { Cross-sectional study } \\
\square \text { Ecological study } \\
\square \text { Systemic review, meta-an } \\
\square \text { Other (specify: }\end{array}$ & ) \\
\hline Background and ob & & \\
\hline Setting & Setting (committee) & $\begin{array}{l}\square \text { Epidemiology and prevention } \\
\square \text { Community } \\
\square \text { EMS } \\
\square \text { Hospital } \\
\square \text { Hypothermia and postresuscitation care } \\
\square \text { Cardiac care resuscitation } \\
\square \text { Pediatric }\end{array}$ \\
\hline & Location & \\
\hline & Duration & \\
\hline Data source & & \\
\hline & Inclusion criteria & \\
\hline Study population & Exclusion criteria & \\
\hline & Matching criteria, if needed & \\
\hline & Main exposure/intervention & \\
\hline - & Other exposure & \\
\hline & Main outcome & \\
\hline & Potential confounders & \\
\hline Optional variables & & \\
\hline Statistical methods & & \\
\hline Study size & & \\
\hline
\end{tabular}


Appendix 2. Study proposal form of experimental study

\begin{tabular}{|c|c|c|}
\hline Item & & Experimental study content \\
\hline \multicolumn{3}{|l|}{ Proposer } \\
\hline \multicolumn{3}{|l|}{ Co-workers } \\
\hline \multicolumn{3}{|l|}{ Institution } \\
\hline Study design & $\begin{array}{l}\square \text { Parallel } \\
\square \text { Factorial } \\
\square \text { Others ( }\end{array}$ & Allocation ratio \\
\hline \multicolumn{3}{|c|}{ Background and Objectives } \\
\hline \multirow[t]{3}{*}{ Setting } & Setting (Committee) & $\begin{array}{l}\square \text { Epidemiology and prevention } \\
\square \text { Community } \\
\square \text { EMS } \\
\square \text { Hospital } \\
\square \text { Hypothermia and postresuscitation care } \\
\square \text { Cardiac care resuscitation } \\
\square \text { Pediatric }\end{array}$ \\
\hline & Location & \\
\hline & Duration & \\
\hline \multirow{3}{*}{ Study population } & Inclusion criteria & \\
\hline & Exclusion criteria & \\
\hline & Matching criteria, if needed & \\
\hline \multicolumn{3}{|l|}{ Interventions } \\
\hline \multicolumn{3}{|l|}{ Statistical methods } \\
\hline Study size & & \\
\hline
\end{tabular}

